Mathematical Modelling and Analysis

Volume 22 Number 3, May 2017, 311-320

https://doi.org/10.3846/13926292.2017.1309329

(c) Vilnius Gediminas Technical University, 2017
Publisher: Taylor\&Francis and VGTU

http://www.tandfonline.com/TMMA

ISSN: $1392-6292$

eISSN: 1648-3510

\title{
A Backward Identification Problem for an Axis-Symmetric Fractional Diffusion Equation
}

\author{
Xiangtuan Xiong and Xiaojun Ma \\ Department of Mathematics, Northwest Normal University \\ Gansu, China \\ E-mail(corresp.): xiongxt@gmail.com \\ E-mail: xiongxt@fudan.edu.cn
}

Received November 4, 2016; revised March 16, 2017; published online May 15, 2017

\begin{abstract}
We consider a backward ill-posed problem for an axis-symmetric fractional diffusion equation which is described in polar coordinates. A closed form solution of the inverse problem is obtained. However, this solution blows up. For numerical stability, a general regularization principle is presented for constructing regularization methods. Several numerical examples are conducted for showing the validity and effectiveness of the proposed methods.
\end{abstract}

Keywords: ill-posedness, regularization, fractional diffusion equation, closed form solution.

AMS Subject Classification: 65R35; 47A52.

\section{Introduction}

Recently, much attention is attracted to the anomalous diffusion phenomena [3]. The forward problems for fractional diffusion equations have been widely investigated. However, inverse problems connected with fractional diffusion are not studied enough in spite of the significance. Since Cheng and Yamamoto [1] opened up a path to the field of the inverse problem for fractional diffusion equations, the work on this topic mushroomed. These inverse problems can be divided into several categories: (1) Numerical differentiation problems,e.g., [10, 16,17], (2) Inverse source problems, e.g., [14,24], (3) Backward time-fractional diffusion problems, e.g., [11, 20,21,22], (4) Inverse coefficient problems, e.g., $[5,9,15]$, (5) Inverse order problems, e.g., [4, 12], (6) Inverse Sturm-Liouville problems, e.g., [6].

The difficulty of inverse problem for fractional diffusion equations lies in the ill-posedness and the fractional derivative. For more details on the inverse problems, the readers can refer to a recent survey [7] and the references therein. However, the works on inverse problems are considered in the Cartesian coordinates. For backward time-fractional diffusion problems, Dou and 
Hon [2] presented a fundamental solution method combined classical regularization methods, Liu [13,22] considered two quasi-reversibility regularization methods. Yang and Liu [23] proposed a Fourier method.

In this article, we consider an axis-symmetric fractional diffusion equation. Let $f(t) \in C[a, b]$ be a time-dependant function, $0<\alpha<1$ is the order of a fractional derivative and the Caputo fractional derivative is defined as follows [18]

$$
{ }_{t} D_{*}^{\alpha} u(x, t)=\frac{1}{\Gamma(1-\alpha)} \int_{0}^{t} \frac{\partial u(x, \eta)}{\partial \eta} \frac{d \eta}{(t-\eta)^{\alpha}} .
$$

A two-parameter Mittag-Leffler function is defined as

$$
E_{\alpha, \beta}=\sum_{k=0}^{\infty} \frac{z^{k}}{\Gamma(\alpha k+\beta)}, \alpha>0, \beta>0, z \in \mathbb{C} .
$$

The forward axis-symmetric fractional diffusion problem now can be expressed as follows:

$$
{ }_{t} D_{*}^{\alpha} u(r, t)=\kappa\left(\frac{\partial^{2} u(r, t)}{\partial r^{2}}+\frac{1}{r} \frac{\partial u(r, t)}{\partial r}\right), 0<r<R, 0<t<T,
$$

subject to the following boundary and initial conditions

$$
\begin{aligned}
& u(R, t)=0,0<t<T, \quad \lim _{r \rightarrow 0} u(r, t) \text { bounded, } 0<t<T, \\
& u(r, 0)=f(r), 0<r<R .
\end{aligned}
$$

Inverse Problem: we want to recover the solution $u(r, t) \in L^{2}((0, R), \rho)$ with $0 \leq t<T$ from the final time data $u(r, T):=g(r) \in L^{2}((0, R), \rho)$ where $L^{2}((0, R), \rho)$ denotes the Hilbert space of squares Lebesgue measurable functions with weight $\rho(r)=r / R^{2}$ defined on $(0, R)$.

The aim of this work is to investigate mathematically and numerically the ill-posedness nature of the backward identification problem. Under the setting of a radial diffusion geometry, the solution is explicitly constructed as an infinite series in terms of the eigenfunctions. Due to the "amplifying factor", small perturbation of the data can lead to large error in the solution. Based on the observation on the ill-posedness nature, we give a general regularization principle for numerical stable solution.

\section{The closed form solution}

First we solve the forward problem by the separation of variables. For this purpose, the analytical solution of problem (1.1)-(1.2) is assumed as

$$
u(r, t)=\sum_{i=1}^{\infty} \varphi_{i}(r) q_{i}(t), i=1,2, \cdots, \infty,
$$

where $\varphi_{i}(r)$ are called eigenfunctions. Substituting equation (2.1) into equation (1.1), we can obtain the Bessel differential equation and fractional differential 
equation. In fact, the eigenfunctions $\varphi_{i}(r)$ are the solutions of Bessel differential equations:

$$
\frac{d^{2} \varphi}{d r^{2}}+\frac{1}{r} \frac{d \varphi}{d r}+\lambda \varphi=0
$$

the boundary conditions are $\lim _{r \rightarrow 0} \varphi(r)$ bounded and $\varphi(R)=0$. And $\lambda$ is the separation constant.

Considering the boundary condition, we can calculate the separation constant $\lambda_{i}=\left(\frac{\mu_{i}}{R}\right)^{2}$, where the $\mu_{i}, i=1,2, \cdots, \infty$ are the positive zeros of the the zero-order Bessel function of the first kind $J_{0}(\mu)$, the eigenfunctions $\varphi_{i}$ are $\varphi_{i}(r)=J_{0}\left(\mu_{i} \frac{r}{R}\right)$ which is a complete orthogonal basis in $L^{2}((0, R), \rho)$.

Now the analytical solution (2.1) of the problem is rewritten as

$$
u(r, t)=\sum_{i=1}^{\infty} q_{i}(t) J_{0}\left(\mu_{i} \frac{r}{R}\right), i=1,2, \cdots, \infty .
$$

First the orthogonality relationship holds

$$
\int_{0}^{1} x J_{0}\left(\mu_{i} x\right) J_{0}\left(\mu_{j} x\right) d x= \begin{cases}0, & i \neq j \\ J_{1}^{2}\left(\mu_{j}\right) / 2, & i=j\end{cases}
$$

i.e.,

$$
\int_{0}^{R} \frac{r}{R^{2}} J_{0}\left(\mu_{i} \frac{r}{R}\right) J_{0}\left(\mu_{j} \frac{r}{R}\right) d r= \begin{cases}0, & i \neq j \\ J_{1}^{2}\left(\mu_{j}\right) / 2, & i=j .\end{cases}
$$

From the above equation, we can easily conclude that the function $q_{i}(t)$ satisfies the following fractional differentiation equation:

$$
{ }_{t} D_{*}^{\alpha} q_{i}(t)=-\kappa\left(\mu_{i} / R\right)^{2} q_{i}(t)
$$

and the initial condition $q_{i}(0)=f_{i}$, where $f_{i}$ is

$$
f_{i}=\frac{2}{R^{2} J_{1}^{2}\left(\mu_{i}\right)} \int_{0}^{R} r J_{0}\left(\mu_{i} \frac{r}{R}\right) f(r) d r .
$$

Solving the fractional differentiation equation, we can easily get the solution

$$
q_{i}(t)=E_{\alpha, 1}\left(-\kappa\left(\mu_{i} / R\right)^{2} t^{\alpha}\right) f_{i}
$$

Now, finally we have the analytic solution for the forward problem (1.1)-(1.2):

$$
u(r, t)=\sum_{i=1}^{\infty} J_{0}\left(\mu_{i} \frac{r}{R}\right) E_{\alpha, 1}\left(-\kappa\left(\frac{\mu_{i}}{R}\right)^{2} t^{\alpha}\right) f_{i}, i=1,2, \cdots, \infty .
$$

Now we turn to solving the inverse problem. From (2.2), we have the equality:

$$
g(r):=u(r, T)=\sum_{i=1}^{\infty} J_{0}\left(\mu_{i} \frac{r}{R}\right) E_{\alpha, 1}\left(-\kappa\left(\frac{\mu_{i}}{R}\right)^{2} T^{\alpha}\right) f_{i}, i=1,2, \cdots, \infty .
$$


Using the orthogonality relationship, we have

$$
g_{i}=E_{\alpha, 1}\left(-\kappa\left(\mu_{i} / R\right)^{2} T^{\alpha}\right) f_{i}, i=1,2, \cdots, \infty,
$$

where $g_{i}=\frac{2}{R^{2} J_{1}^{2}\left(\mu_{i}\right)} \int_{0}^{R} r J_{0}\left(\mu_{i} \frac{r}{R}\right) g(r) d r$.

Therefore, the solution for the inverse problem is given by

$$
u(r, t)=\sum_{i=1}^{\infty} J_{0}\left(\mu_{i} \frac{r}{R}\right) \frac{E_{\alpha, 1}\left(-\kappa\left(\frac{\mu_{i}}{R}\right)^{2} t^{\alpha}\right)}{E_{\alpha, 1}\left(-\kappa\left(\frac{\mu_{i}}{R}\right)^{2} T^{\alpha}\right)} g_{i}, i=1,2, \cdots, \infty .
$$

Remark 1. If $\alpha=1$, in fact $E_{1,1}\left(-\kappa\left(\frac{\mu_{i}}{R}\right)^{2} t^{\alpha}\right)=\exp \left(-\kappa\left(\frac{\mu_{i}}{R}\right)^{2} t\right)$ and $E_{1,1}\left(-\kappa\left(\frac{\mu_{i}}{R}\right)^{2} T^{\alpha}\right)=\exp \left(-\kappa\left(\frac{\mu_{i}}{R}\right)^{2} T\right)$, then the solution for the inverse problem is given by

$$
u(r, t)=\sum_{i=1}^{\infty} J_{0}\left(\mu_{i} \frac{r}{R}\right) \exp \left(\kappa\left(\frac{\mu_{i}}{R}\right)^{2}(T-t)\right) g_{i}, i=1,2, \cdots, \infty .
$$

Since $\mu_{i} \rightarrow \infty$ as $i \rightarrow \infty$, we have $\exp \left(\kappa\left(\frac{\mu_{i}}{R}\right)^{2}(T-t)\right) \rightarrow \infty$ for $T>t$. Therefore the backward identification problem is an ill-posed problem.

Remark 2. If we consider the similar problem which is formulated in the case of Cartesian coordinate:

$$
{ }_{t} D_{*}^{\alpha} u(x, t)=u_{x x}(x, t), 0<x<R, 0<t<T
$$

with boundary conditions $u(0, t)=u(R, t)=0$ and final condition $u(x, T)=$ $g(x)$. The solution for the inverse problem is given by

$$
u(x, t)=\sum_{n=1}^{\infty} \sin \left(\frac{n \pi}{R} x\right) \frac{E_{\alpha, 1}\left(-\kappa(n \pi / R)^{2} t^{\alpha}\right)}{E_{\alpha, 1}\left(-\kappa(n \pi / R)^{2} T^{\alpha}\right)} g_{n}, n=1,2, \cdots, \infty,
$$

where $g_{n}$ is the sine Fourier coefficients. Comparing this expression with (2.4), we can see that the problems in the two cases have almost the same ill-posedness.

In this paper, we are more interested in finding the inverse solution $u(r, 0)$ in (2.4). Here noting that $E_{\alpha, 1}(0)=1$ we write the inverse solution as

$$
u(r, 0)=\sum_{i=1}^{\infty} J_{0}\left(\mu_{i} \frac{r}{R}\right) \frac{1}{E_{\alpha, 1}\left(-\kappa\left(\frac{\mu_{i}}{R}\right)^{2} T^{\alpha}\right)} g_{i}, i=1,2, \cdots, \infty .
$$

In order to show the instability of the solution, we need a lemma from [13].

Lemma 1. Assume that $0<\alpha_{0}<\alpha_{1}<1$, then there exist constants $C_{2}>$ $C_{1}>0$ depending only on $\alpha_{0}, \alpha_{1}$, such that

$$
\frac{C_{1}}{\Gamma(1-\alpha)} \frac{1}{1-x} \leq E_{\alpha, 1}(x) \leq \frac{C_{2}}{\Gamma(1-\alpha)} \frac{1}{1-x}, \forall x \leq 0,
$$

these estimates are uniform for all $\alpha \in\left[\alpha_{0}, \alpha_{1}\right]$. 
From Lemma 1, we can conclude that

$$
\frac{\Gamma(1-\alpha)}{C_{2}}\left(1+\kappa\left(\frac{\mu_{i}}{R}\right)^{2} T^{\alpha}\right) \leq \frac{1}{E_{\alpha, 1}\left(-\kappa\left(\frac{\mu_{i}}{R}\right)^{2} T^{\alpha}\right)} \leq \frac{\Gamma(1-\alpha)}{C_{1}}\left(1+\kappa\left(\frac{\mu_{i}}{R}\right)^{2} T^{\alpha}\right) .
$$

Because $\mu_{i} \rightarrow \infty$ as $i \rightarrow \infty$, we have

$$
\frac{1}{E_{\alpha, 1}\left(-\kappa\left(\frac{\mu_{i}}{R}\right)^{2} T^{\alpha}\right)}=O\left(\kappa\left(\frac{\mu_{i}}{R}\right)^{2} T^{\alpha}\right)
$$

with a fixed $\alpha$ which is far away from 1 .

Therefore, from (2.5) we can see that a small perturbation in the data $g$ can cause a large change in the solution $u(r, 0)$. In the case of fractional derivative, the backward identification problem is mildly ill-posed. From above analysis, we know that the ill-posedness of the backward identification problem is caused by the factor $\frac{1}{E_{\alpha, 1}\left(-\kappa\left(\frac{\mu_{i}}{R}\right)^{2} T^{\alpha}\right)}$. We call $K\left(r, \mu_{i}\right)=\frac{1}{E_{\alpha, 1}\left(-\kappa\left(\frac{\mu_{i}}{R}\right)^{2} T^{\alpha}\right)}$ amplifying factor of the problem.

\section{A regularization principle and its applications}

Motivated by the filter methods [19], we can approximate the amplifying factor stably and thus stabilize the ill-posed problem. By introducing a regularization parameter $\tilde{\alpha}$, we propose a general principle for constructing the stabilized amplifying factor $K_{\tilde{\alpha}}\left(r, \mu_{i}\right)$ which approaches the amplifying factor $K\left(r, \mu_{i}\right)$ :

A general principle for constructing $K_{\tilde{\alpha}}\left(r, \mu_{i}\right)$ :

(1) for every $\mu_{i}, \lim _{\tilde{\alpha} \rightarrow 0} K_{\tilde{\alpha}}\left(r, \mu_{i}\right)=K\left(r, \mu_{i}\right)$;

(2) for every $\tilde{\alpha}>0$, there exists a constant $C(\tilde{\alpha})$ which is dependent on $\tilde{\alpha}$ such that $\left|K_{\tilde{\alpha}}\left(r, \mu_{i}\right)\right| \leq C(\tilde{\alpha})$ for all $\mu_{i}$;

(3) there exists constant $c>0$ such that $\left|K_{\tilde{\alpha}}\left(r, \mu_{i}\right)\right| \leq c K\left(r, \mu_{i}\right)$ for all $\tilde{\alpha}$ and $\mu_{i}$.

The first condition guarantees the approximation property. The last two conditions guarantee the stabilization of the approximation problem.

Based on the principle, we can construct several specific regularization methods. For examples, we list some of them:

Method 1. The first stabilized amplifying factor $K_{\alpha}\left(r, \mu_{i}\right)$ is given by

$$
K_{\tilde{\alpha}}\left(r, \mu_{i}\right)=\frac{1}{E_{\alpha, 1}\left(-\kappa\left(\mu_{i} / R\right)^{2} T^{\alpha}\right)} \chi_{\frac{1}{\alpha}}
$$

where $\chi_{\frac{1}{\tilde{\alpha}}}$ is the characteristic function, i.e., $\chi_{\frac{1}{\tilde{\alpha}}}=1$ if $\mu_{i} \leq \frac{1}{\tilde{\alpha}}$ and $\chi_{\frac{1}{\tilde{\alpha}}}=0$ if $\mu_{i}>\frac{1}{\tilde{\alpha}}$.

Method 2. The second stabilized amplifying factor $K_{\tilde{\alpha}}\left(r, \mu_{i}\right)$ is given by

$$
K_{\tilde{\alpha}}\left(r, \mu_{i}\right)=\frac{E_{\alpha, 1}\left(-\kappa\left(\mu_{i} / R\right)^{2} T^{\alpha}\right)}{E_{\alpha, 1}^{2}\left(-\kappa\left(\mu_{i} / R\right)^{2} T^{\alpha}\right)+\tilde{\alpha}} .
$$

Correspondingly, we write these two regularization solutions as follows: 
Solution 1. The first regularization solution $u_{\tilde{\alpha}}(r, 0)$ is given by

$$
u_{\tilde{\alpha}}(r, 0)=\sum_{i=1}^{\infty} J_{0}\left(\mu_{i} \frac{r}{R}\right) \frac{1}{E_{\alpha, 1}\left(-\kappa\left(\mu_{i} / R\right)^{2} T^{\alpha}\right)} \chi_{\frac{1}{\tilde{\alpha}}} g_{i} .
$$

This method is known as spectral cut-off method. In practical computation, we use

$$
u_{M}(r, 0)=\sum_{i=1}^{M} J_{0}\left(\mu_{i} \frac{r}{R}\right) \frac{1}{E_{\alpha, 1}\left(-\kappa\left(\mu_{i} / R\right)^{2} T^{\alpha}\right)} g_{i},
$$

where $M$ is the regularization parameter.

Solution 2. The second regularization solution $u_{\tilde{\alpha}}(r, 0)$ is given by

$$
u_{\tilde{\alpha}}(r, 0)=\sum_{i=1}^{\infty} J_{0}\left(\mu_{i} \frac{r}{R}\right) \frac{E_{\alpha, 1}\left(-\kappa\left(\mu_{i} / R\right)^{2} T^{\alpha}\right)}{E_{\alpha, 1}^{2}\left(-\kappa\left(\mu_{i} / R\right)^{2} T^{\alpha}\right)+\tilde{\alpha}} g_{i} .
$$

Remark 3. Motivated by the recently-developed fractional Tikhonov regularization methods [8], it is interesting to give some fractional regularization methods, e,g. the fractional Tikhonov method. This method is given by modifying the amplifying factor as

$$
K_{\tilde{\alpha}}\left(r, \mu_{i}\right)=\frac{E_{\alpha, 1}\left(-\kappa\left(\mu_{i} / R\right)^{2} T^{\alpha}\right)}{E_{\alpha, 1}^{\gamma}\left(-\kappa\left(\mu_{i} / R\right)^{2} T^{\alpha}\right)+\tilde{\alpha}}
$$

with $1 \leq \gamma \leq 2$

\section{Numerical examples}

Now we give some numerical examples to test the proposed regularization principle. In this section, M1 and M2 represent Method 1, Method 2 , respectively. First we solve the forward problem with some specified function $f(r)$ to simulate the final value $u(r, T):=g(r)$ by $(2.2)$, then we add artificial random noise to $g(r)$ for generating the noisy data $g_{\delta}(r)$.

To avoid the "inverse crime", we use two different grid configurations of $f(r)$ for solving forward and backward problems. For the forward problem, we specify the value $f(r)$ at a coarse grid $\left\{r_{j}\right\}, j=0, \ldots, M_{1}$ to get $g(r)$ at the coarse grid. After generating the noisy data $g_{\delta}(r)$, by interpolation, we can get the values of $g_{\delta}(r)$ at a finer gird $\left\{r_{j}\right\}, j=0, \ldots, M_{2}$. Then we solve the backward problem by regularization methods to get the values of $f(r)$ at the finer gird $\left\{r_{j}\right\}, j=0, \ldots, M_{2}$.

In the examples, we take $R=1, T=1, M_{1}=100, M_{2}=400$. We replace $\infty$ in the solutions with a large integer $\tilde{M}$ (i.e., we truncate the series) in M1, M2 and (2.2). However in M2, the integer $M$ plays the role of regularization parameter. The discrete version of noisy data $g(r)$ is generated as follows:

$$
g_{j}^{\delta}=g_{j}+\max g_{j} \delta \operatorname{rand}(\operatorname{length}(g))_{j}, \quad j=1, \ldots, M_{2},
$$

where $g_{j}$ are the exact data and $\max g_{j}$ denotes the maximum of the exact data $g_{j}, \operatorname{rand}(\operatorname{length}(g))_{j}$ is a random number, $\delta$ is the noise level. 
Example 1. Consider the initial data $f(r)=e^{-r^{2}} \sin (2 \pi r)$. Figure 1 (a) shows the results for the computed $g(r)$ and Figure 1(b) shows the computed $f(r)$ by (2.5) with $\alpha=0.5$ and $\delta=1 \%$. We can see that the solution given by (2.5) is unstable solution for computation because the ill-posedness of problem. Therefore the regularization method is necessary.

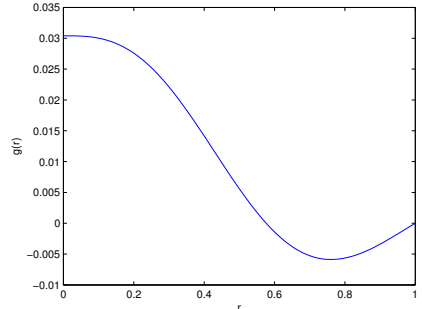

(a)

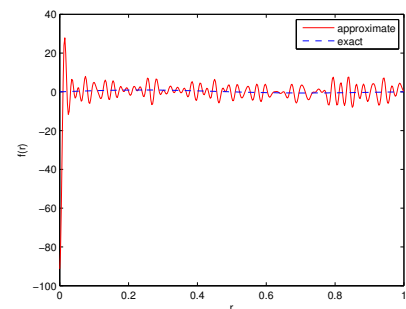

(b)

Figure 1. Example 1: (a) the input data $g(t)$, (b) the direct computational result with noisy data

When $\alpha=0.5$ and $\delta=1 \%$, we use the regularization methods M1 and M2 to construct the approximation. The results are displayed in Figure 2 (a) and (b). From them it is easy to see that the presented methods are effective.

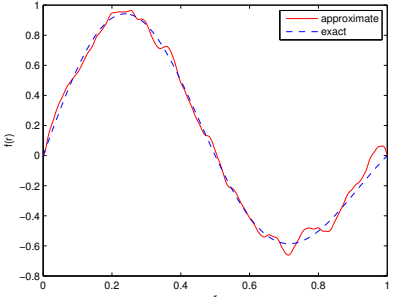

(a)

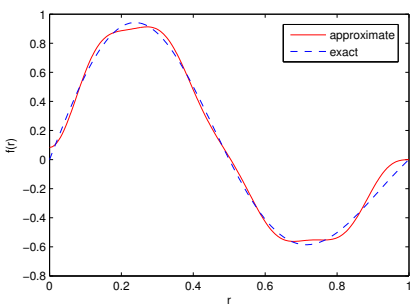

(b)

Figure 2. Example 1: (a) results by M2 with $\tilde{\alpha}=1 * 10^{-7}$, (b) Result by M1 with $M=10$.

Example 2. In Example 1, the exact solution are too smooth. In this example, we consider a hard example, i.e., the initial data is given by

$$
f(t)= \begin{cases}1, & \text { if } 0.25 \leq t \leq 0.75 \\ 0, & \text { else }\end{cases}
$$

We find the methods are still effective. First Figure 3 (a) and (b) show the reconstruction results for M1 with a fixed fractional order $\alpha=0.1, M=10$ and different noise levels. From these figures we see that the results worsen as the noise becomes larger. 


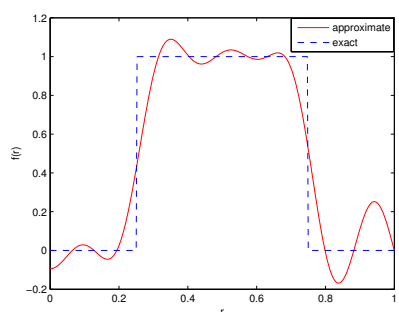

(a)

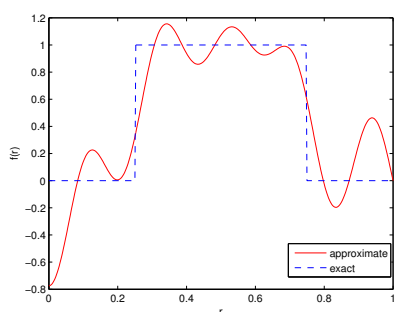

(b)

Figure 3. Example 2. Method 1 with different noisy levels: (a) result by M1 with $\delta=0.01, \quad$ (b) result by M1 with $\delta=0.03$.

Figure 4 (a), (b) and (c) show the results with different fractional orders $\alpha$ with a fixed noise level $\delta=1 \%, \tilde{\alpha}=1 * 10^{-6}$. The larger $\alpha$ is, the worse reconstruction result is. This is because the degree of ill-posedness increases as the fractional order $\alpha$ increases. And the degree of ill-posedness becomes the largest at $\alpha=1$.

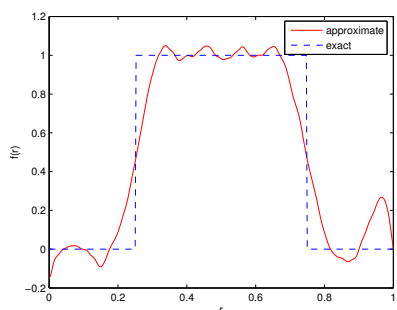

(a)

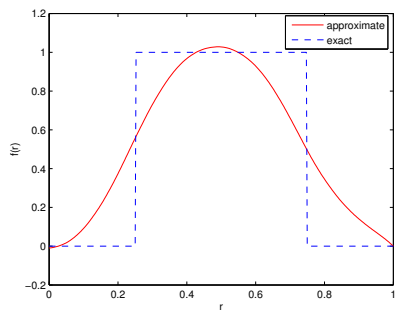

(c)

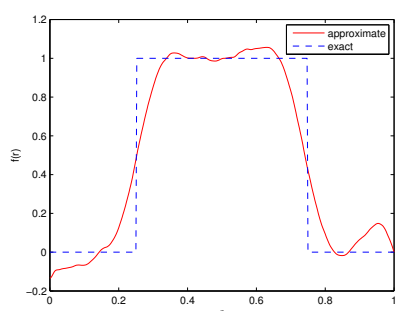

(b)

Figure 4. Example 2 (a) M2 with fractional order $\alpha=0.1$, (b) M2 with different fractional order $\alpha=0.5$, (c) M2 with different fractional order $\alpha=0.9$.

\section{Acknowledgements}

The authors would like to thank Prof. I.Podlubny for providing the program on Mittag-Leffler function on the web http://www.mathworks.com. 
The authors would like to thank the reviewers for their important comments.

The research was partially supported by a grant from the National Natural Science Foundation of China (No. 11661072), and the Natural Science Foundation of Gansu Province, China (No. 145RJZA037 ).

\section{References}

[1] J. Cheng, J. Nakagawa, M. Yamamoto and T. Yamazaki. Uniqueness in an inverse problem for a one-dimensional fractional diffusion equation. Inverse Problems, 25(11):115002, 2009.

[2] F.F. Dou and Y.C. Hon. Numerical computation for backward time-fractional diffusion equation. Engineering Analysis with Boundary Elements, 40:138-146, 2014. https://doi.org/10.1016/j.enganabound.2013.12.001.

[3] R. Gorenflo and F. Mainardi. Random walk models for space fractional diffusion processes. Fractional Calculus and Applied Analysis, 1(2):167-191, 1998.

[4] Y. Hatano, J. Nakagawa, S. Wang and M. Yamamoto. Determination of order in fractional diffusion equation. Journal of Math-for-Industry, 5(A):51-57, 2013.

[5] B. Jin and W. Rundell. An inverse problem for a one-dimensional time-fractional diffusion problem. Inverse Problems, 28(7):075010, 2012.

[6] B. Jin and W. Rundell. An inverse Sturm-Liouville problem with a fractional derivative. Journal of Computational Physics, 231(14):4954-4966, 2012. https://doi.org/10.1016/j.jcp.2012.04.005.

[7] B. Jin and W. Rundell. A tutorial on inverse problems for anomalous diffusion processes. Inverse Problems, 31(3):035003, 2015.

[8] E. Klann and R. Ramlau. Regularization by fractional filter methods and data smoothing. Inverse Problems, 24(2):045005, 2008.

[9] G. Li, D. Zhang, X. Jia and M. Yamamoto. Simultaneous inversion for the spacedependent diffusion coefficient and the fractional order in the time-fractional diffusion equation. Inverse Problems, 29(6):065014, 2013.

[10] M. Li, Y. Wang and L. Ling. Numerical Caputo differentiation by radial basis functions. Journal of Scientific Computing, 62(1):300-315, 2015. https://doi.org/10.1007/s10915-014-9857-6.

[11] M. Li and X.T. Xiong. On a fractional backward heat conduction problem: Application to deblurring. Computers and Mathematics with Applications, 64(8):2594-2602, 2012. https://doi.org/10.1016/j.camwa.2012.07.003.

[12] Z. Li and M. Yamamoto. Uniqueness for inverse problems of determining orders of multi-term time-fractional derivatives of diffusion equation. Applicable Analysis, 94(3):570-579, 2015. https://doi.org/10.1080/00036811.2014.926335.

[13] J.J. Liu and M. Yamamoto. A backward problem for the timefractional diffusion equation. Applicable Analysis, 89(11):1769-1788, 2010. https://doi.org/10.1080/00036810903479731.

[14] Y. Luchko, W. Rundell, M. Yamamoto and L. Zuo. Uniqueness and reconstruction of an unknown semilinear term in a time-fractional reaction-diffusion equation. Inverse Problems, 29(6):065019, 2013.

[15] L. Miller and M. Yamamoto. Coefficient inverse problem for a fractional diffusion equation. Inverse Problems, 29(7):075013, 2013. 
[16] D.A. Murio. On the stable numerical evaluation of Caputo fractional derivatives. Computers and Mathematics with Applications, 51(9):1539-1550, 2006. https://doi.org/10.1016/j.camwa.2005.11.037.

[17] L. Pandolfi. A Lavrent'ev-type approach to the on-line computation of Caputo fractional derivatives. Inverse Problems, 24(1):015014, 2008.

[18] I. Podlubny. Fractional Differential Equations. Academic Press, San Diego, 1999.

[19] Z. Qian, B.Y.C. Hon and X.T. Xiong. Numerical solution of two-dimensional radially symmetric inverse heat conduction problem. Journal of Inverse and Illposed Problems, 23(2):121-134, 2015. https://doi.org/10.1515/jiip-2012-0102.

[20] K. Sakamoto and M. Yamamoto. Inital value/boudnary value problems for fractional diffusion-wave equations and applications to some inverse problems. Journal of Mathematical Analysis and Applications, 382(1):426-447, 2011. https://doi.org/10.1016/j.jmaa.2011.04.058.

[21] L. Wang and J. Liu. Total variation regularization for a backward time-fractional diffusion problem. Inverse Problems, 29(11):115013, 2013.

[22] M. Yang and J. Liu. Solving a final value fractional diffusion problem by boundary condition regularization. Applied Numerical Mathematics, 66:45-58, 2013. https://doi.org/10.1016/j.apnum.2012.11.009.

[23] M. Yang and J. Liu. Fourier regularization for a final value timefractional diffusion equation. Applicable Analysis, 94(7):1508-1526, 2015. https://doi.org/10.1080/00036811.2014.936402.

[24] Y. Zhang and X. Xu. Inverse source problem for a fractional diffusion equation. Inverse Problems, 27(3):035010, 2011. 